Research Article

\title{
Existence and Uniqueness of Fixed Points of Generalized F-Contraction Mappings
}

\author{
A. P. Farajzadeh $\mathbb{D}^{1},{ }^{1}$ M. Delfani $\left(\mathbb{D},{ }^{1}\right.$ and Y. H. Wang $(\mathbb{D})^{2}$ \\ ${ }^{1}$ Department of Mathematics, Razi University, Kermanshah 67149, Iran \\ ${ }^{2}$ Department of Mathematics, Zhejiang Normal University, Jinhua, Zhejiang 321004, China
}

Correspondence should be addressed to Y. H. Wang; yhwang@zjnu.cn

Received 22 November 2020; Revised 6 December 2020; Accepted 12 January 2021; Published 29 January 2021

Academic Editor: Ljubisa Kocinac

Copyright (C) 2021 A. P. Farajzadeh et al. This is an open access article distributed under the Creative Commons Attribution License, which permits unrestricted use, distribution, and reproduction in any medium, provided the original work is properly cited.

\begin{abstract}
The newest generalization of the Banach contraction through the notions of the generalized F-contraction, simulation function, and admissible function is introduced. The existence and uniqueness of fixed points for a self-mapping on complete metric spaces by the new constructed contraction are investigated. The results of this article can be viewed as an improvement of the main results given in the references.
\end{abstract}

\section{Introduction and Preliminaries}

In 1922, Banach proved the following famous and fundamental result in fixed-point theory [1]. Let $(X, d)$ be a complete metric space. Let $T$ be a contractive mapping on $X$; that is, there exists $q \in[0,1)$ satisfying

$$
d(T x, T y) \leq q . d(x, y), \forall x, y \in X .
$$

Then, there exists a unique fixed point $x_{0} \in X$ of $T$. This theorem, which is called the Banach contraction principle that is a forceful tool in nonlinear analysis [9-14] and fixed-point theory, is a fascinating subject, with an enormous number of algorithms and applications in various fields of mathematics, see, e.g., [15-18]. This principle has been generalized in different directions by various researchers. One of them is the following theorem that is presented by Bryant.

Theorem 1 (see [2]). If $f$ is a mapping of a complete metric space into itself and if, for some positive integer $k, f^{k}$ is a contraction, then $f$ has a unique fixed point.

It is obvious that $f^{k}$ is continuous but there are examples that show it cannot imply the continuity of $f$ and so Theorem 1 is a real extension of the Banach principle.
In 1969, Sehgal [19] proved the following interesting generalization of Theorem 1 .

Theorem 2 (see [19]). Let $(X, d)$ be a complete metric space, $q \in[0,1)$, and $T: X \longrightarrow X$ be a continuous mapping. If for each $x \in X$ there exists a positive integer $k=k(x)$ such that

$$
d\left(T^{k(x)} x, T^{k(x)} y\right) \leq q d(x, y),
$$

for all $y \in X$, then $T$ has a unique fixed point $u \in X$. Moreover, for any $x \in X, u=\lim _{n \longrightarrow \infty} T^{n} x$.

Several researchers are interested to generalize Banach contraction. Here, we state two of them. Wardowski [8] generalized the Banach contraction as follows.

Definition 1 (see [8]). Let $(X, d)$ be a metric space. The mapping $T: X \longrightarrow X$ is called an F-contraction, if there exist $F \in \mathscr{F}$ and $\tau>0$ such that, for all $x, y \in X$,

$$
d(T x, T y)>0 \Rightarrow \tau+F(d(T x, T y)) \leq F(d(x, y)),
$$

where $F:(0, \infty) \longrightarrow \mathbb{R}$ is strictly increasing $\lim _{n \rightarrow \infty} F\left(\alpha_{n}\right)=$ $-\infty$ iff $\lim _{n \longrightarrow \infty} \alpha_{n}=0$ and there exists a number $k \in(0,1)$ such that $\lim _{\alpha \longrightarrow 0^{+}} \alpha^{k} F(\alpha)=-\infty$. 
Notation. The family of all functions $F:(0,+\infty) \longrightarrow \mathbb{R}$ is denoted by $\mathscr{F}$ (see [8]) if $F$ satisfies the following conditions:

(F1) $F$ is strictly increasing

(F2) for every sequence $\left\{\alpha_{n}\right\}$ in $(0,+\infty)$, we have $\lim _{n \longrightarrow \infty} F\left(\alpha_{n}\right)=-\infty$ iff $\lim _{n \longrightarrow \infty} \alpha_{n}=0$

(F3) there exists a number $k \in(0,1)$ such that $\lim _{\alpha \longrightarrow 0^{+}} \alpha^{k} F(\alpha)=-\infty$

The collection of all functions $F:(0,+\infty) \longrightarrow \mathbb{R}$ is denoted by $\mathscr{G}$ ( [20]) if $F$ satisfies the following conditions:

(G1) $F$ is strictly increasing

(G2) there exists a sequence $\left\{\alpha_{n}\right\}$ in $(0,+\infty)$ such that $\lim _{n \rightarrow \infty} F\left(\alpha_{n}\right)=-\infty$, or inf $F=-\infty$

(G3) $F$ is a continuous mapping

Another way to generalize the Banach contraction is through the following notion.

Definition 2 (see $[3,21])$. Let $\zeta:[0, \infty) \times[0, \infty) \longrightarrow \mathbb{R}$ be a mapping, then $\zeta$ is called a simulation function if it satisfies the following conditions:

$(\zeta 1) \zeta(0,0)=0$

$(\zeta 2) \zeta(t, s)<s-t$ for all $t, s>0$

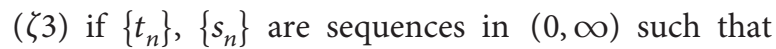
$\lim _{n \longrightarrow \infty} t_{n}=\lim _{n \longrightarrow \infty} s_{n}>0$ and $t_{n}<s_{n}$ for all $n \in \mathbb{N}$, then

$$
\limsup _{n \longrightarrow \infty} \zeta\left(t_{n}, s_{n}\right)<0
$$

We denote the set of all simulation functions by $\mathscr{Z}$.

Ozturk [4], by using the simulation function and Wardowski [8] idea, extended Theorem 2 as follows.

Theorem 3 (see [4]). Let $(X, d)$ be a complete metric space and $T: X \longrightarrow X$ a mapping which satisfies the condition: If there exist $f \in \mathscr{F}$ and $\tau>0$ such that for each $x \in X$ there is a positive integer $n(x)$ such that for all $y \in X$,

$$
\begin{aligned}
d\left(T^{n(x)}(x), T^{n(x)}(y)\right)> & 0 \Rightarrow \zeta(F(d(x, y)), \tau \\
& \left.+F\left(d\left(T^{n(x)}(x), T^{n(x)}(y)\right)\right)\right) \geq 0
\end{aligned}
$$

then $T$ has a unique fixed point $z \in X$ and $T^{n}\left(x_{0}\right) \longrightarrow z$ for each $x_{0} \in X$, as $n \longrightarrow \infty$.

The first aim of this paper is to generalize Theorem 2 by introducing a more general contraction type mapping through the notions of the generalized F-contraction, simulation function, and admissible function. Then, by the new constructed contraction and suitable conditions, the existence and uniqueness of fixed points are investigated.

The following definitions and preliminary results are needed in the next section.
Definition 3 (see $[6,22])$. Let $\alpha: X \times X \longrightarrow(0,+\infty)$ be a given mapping. The mapping $T: X \longrightarrow X$ is said to be an $\alpha$-admissible, whenever $\alpha(T x, T y) \geq 1$ provided $\alpha(x, y) \geq 1$ and $x, y \in X$.

Definition 4 (see [23]). An $\alpha$-admissible map $T$ is said to have the K-property, while for each sequence $\left\{x_{n}\right\} \subseteq X$ with $\alpha\left(x_{n}, x_{n+1}\right) \geq 1$ for all $n \in \mathbb{N}_{0}$, the nonnegative integer numbers, there exists a positive integer number $k$ such that $\alpha\left(T x_{n}, T x_{m}\right) \geq 1$, for all $m>n \geq k$.

Lemma 1 (see [5]). Let $F:(0,+\infty) \longrightarrow \mathbb{R}$ be an increasing function and $\left\{\alpha_{n}\right\}$ be a sequence of positive real numbers. Then, the following holds:
(a) if $\lim _{n \longrightarrow \infty} F\left(\alpha_{n}\right)=-\infty$, then $\lim _{n \longrightarrow \infty} \alpha_{n}=0$
(b) if inf $F=-\infty$ and $\lim _{n \rightarrow \infty} \alpha_{n}=0$, then $\lim _{n \longrightarrow \infty}$ $F\left(\alpha_{n}\right)=-\infty$

Lemma 2 (see [24]). Let $(X, d)$ be a metric space and $\left\{x_{n}\right\}$ be a sequence in $X$ such that $\lim _{n \rightarrow \infty} d\left(x_{n}, x_{n+1}\right)=0$. If $\left\{x_{n}\right\}$ is not a Cauchy sequence, then there exist $\varepsilon>0$ and two sequences of positive integers $\left\{n_{k}\right\}$ and $\left\{m_{k}\right\}$ with $n_{k}>m_{k}>k$ such that $d\left(x_{m_{k}}, x_{n_{k}}\right)>\varepsilon, d\left(x_{m_{k}}, x_{n_{k}-1}\right)<\varepsilon$, and
(1) $\lim _{k \longrightarrow \infty} d\left(x_{m_{k}}, x_{n_{k}}\right)=\varepsilon$
(2) $\lim _{k \longrightarrow \infty} d\left(x_{m_{k}-1}, x_{n_{k}}\right)=\varepsilon$
(3) $\lim _{k \longrightarrow \infty} d\left(x_{m_{k}}, x_{n_{k}+1}\right)=\varepsilon$
(4) $\lim _{k \longrightarrow \infty} d\left(x_{m_{k}-1}, x_{n_{k}+1}\right)=\varepsilon$

\section{Main Results}

In this section, the main achievements of this article are presented. The existence and uniqueness of fixed points of the self-mappings on complete metric spaces satisfying the generalized F-contraction (relation (6) of the following theorem) with suitable assumptions are established by the first theorem. The second theorem can be viewed as a generalized version of Suzuki's theorem given in [21]. Of course it ensures existence of fixed points for self-mappings under suitable hypothesis.

Theorem 4 Let $(X, d)$ be a complete metric space and $\alpha: X \times X \longrightarrow(0,+\infty)$ be a symmetric function, where $\alpha(x, y) \geq 1$ and $T: X \longrightarrow X$ be a continuous mapping which satisfies the condition: if there exist $F \in \mathscr{F}, \tau>0$, $L \geq 0$, and simulation function $\zeta$ such that for all $x \in X$ there is a positive integer $n(x)$ such that for all $y \in X$ and $d\left(T^{n(x)}(x), T^{n(x)}(y)\right)>0$,

$$
\zeta\left(\tau+\alpha(x, y) F\left(d\left(T^{n(x)} x, T^{n(x)} y\right)\right), F\left(m(x, y)+\mathrm{LN}_{1}(x, y)\right)\right) \geq 0,
$$

where 


$$
\begin{aligned}
& m(x, y)=\max \left\{d(x, y), d\left(x, T^{n(x)} x\right), d\left(y, T^{n(x)} y\right), \frac{d\left(x, T^{n(x)} y\right)+d\left(y, T^{n(x)} x\right)}{2}\right\}, \\
& N_{1}(x, y)=\min \left\{d\left(x, T^{n(x)} x\right), d\left(x, T^{n(x)} y\right), d\left(y, T^{n(x)} x\right)\right\}, \\
& \quad T x_{i_{0}}=T\left(T^{n_{i_{0}}} x_{i_{0}}\right)=T^{n_{i_{0}}}\left(T x_{i_{0}}\right) .
\end{aligned}
$$

then $T$ has a unique fixed point.

Proof. We shall built a recursive sequence $\left\{x_{k}\right\}$ as follows: for the chosen arbitrary point $x_{0} \in X$ with $n_{0}=n\left(x_{0}\right)$, we set $x_{1}=T^{n_{0}} x_{0}$ and inductively we get $x_{i+1}=T^{n_{i}} x_{i}$ with $n_{i}=n\left(x_{i}\right)$.

We assert that $x_{i} \neq x_{i+1}$ for all $i \in \mathbb{N}_{0}$. Suppose, on the contrary, there exists $i_{0} \in N_{0}$ such that $x_{i_{0}}=x_{i_{0}+1}=T^{n_{i 0}} x_{i_{0}}$. Then, $x_{i_{0}}$ turns to be a fixed point of $T^{n_{i_{0}}}$. On the other hand,
Thus, $T x_{i_{0}}$ form a fixed point of $T^{n_{i_{0}}}$. If $T x_{i_{0}}=x_{i_{0}}$, then we conclude that $T$ has a fixed point and that terminate the proof. Suppose, on the contrary, that $T x_{i_{0}} \neq x_{i_{0}}$ and hence $d\left(T^{n_{i_{0}}}\left(T x_{i_{0}}\right), T^{n_{i_{0}}}\left(x_{i_{0}}\right)\right)>0$. Then, by (6), we have

$$
\begin{aligned}
0 & \leq\left(\tau+\alpha\left(x_{i_{0}}, T x_{i_{0}}\right) F\left(d\left(T^{n_{i_{0}}} x_{i_{0}}, T^{n_{i_{0}}} T x_{i_{0}}\right)\right), F\left(m\left(x_{i_{0}}, T x_{i_{0}}\right)+\mathrm{LN}_{1}\left(x_{i_{0}}, T x_{i_{0}}\right)\right)\right), \\
& \leq F\left(m\left(x_{i_{0}}, T x_{i_{0}}\right)+\mathrm{LN}_{1}\left(x_{i_{0}}, T x_{i_{0}}\right)\right)-\left(\tau+\alpha\left(x_{i_{0}}, T x_{i_{0}}\right) F\left(d\left(T^{n_{i_{0}}} x_{i_{0}}, T^{n_{i_{0}}} T x_{i_{0}}\right)\right)\right) .
\end{aligned}
$$

Hence,

However,

$$
\begin{aligned}
\tau+F\left(d\left(x_{i_{0}}, T x_{i_{0}}\right)\right) & =\tau+F\left(d\left(T^{n_{i_{0}}} x_{i_{0}}, T^{n_{i_{0}}} T x_{i_{0}}\right)\right) \\
& \leq \tau+\alpha\left(x_{i_{0}}, T x_{i_{0}}\right) F\left(d\left(T^{n_{i_{0}}} x_{i_{0}}, T^{n_{i_{0}}} T x_{i_{0}}\right)\right) \\
& \leq F\left(m\left(x_{i_{0}}, T x_{i_{0}}\right)+\operatorname{LN}_{1}\left(x_{i_{0}}, T x_{i_{0}}\right)\right) .
\end{aligned}
$$

$$
\begin{aligned}
m\left(x_{i_{0}}, T x_{i_{0}}\right) & =\max \left\{d\left(x_{i_{0}}, T x_{i_{0}}\right), d\left(x_{i_{0}}, T^{n_{i_{0}}} x_{i_{0}}\right), d\left(T x_{i_{0}}, T^{n_{i_{0}}} T x_{i_{0}}\right), \frac{d\left(x_{i_{0}}, T^{n_{i_{0}}} T x_{i_{0}}\right)+d\left(T x_{i_{0}}, T^{n_{i_{0}}} x_{i_{0}}\right)}{2}\right\}=\left\{d\left(x_{i_{0}}, T x_{i_{0}}\right)\right\}, \\
N_{1} & =\min \left\{d\left(x_{i_{0}}, T^{n_{i_{0}}} x_{i_{0}}\right), d\left(x_{i_{0}}, T^{n_{i_{0}}} T x_{i_{0}}\right), d\left(T x_{i_{0}}, T^{n_{i_{0}}} x_{i_{0}}\right)\right\}=0 .
\end{aligned}
$$

Therefore,

$$
\tau+F\left(d\left(x_{i_{0}}, T x_{i_{0}}\right)\right) \leq F\left(d\left(x_{i_{0}}, T x_{i_{0}}\right)\right) .
$$

So, $\tau \leq 0$, which is a contradiction. Consequently, we deduce that for all $i \in \mathbb{N}_{0}, x_{i} \neq x_{i+1}$. Then, $d\left(x_{i+1}, x_{i}\right)>0$, by (6),

$$
\begin{aligned}
\tau+F\left(d\left(x_{i+1}, x_{i+2}\right)\right) & =\tau+F\left(d\left(T^{n_{i}} x_{i}, T^{n_{i}} x_{i+1}\right)\right. \\
& \leq \tau+\alpha\left(x_{i}, x_{i+1}\right) F\left(d\left(T^{n_{i}} x_{i}, T^{n_{i}} x_{i+1}\right)\right. \\
& \leq F\left(m\left(x_{i}, x_{i+1}\right)+\operatorname{LN}_{1}\left(x_{i}, x_{i+1}\right)\right) \\
& \leq F\left(m\left(x_{i}, x_{i+1}\right)+L d\left(x_{i+1}, x_{i+1}\right)\right) \\
& =F\left(m\left(x_{i}, x_{i+1}\right)\right) .
\end{aligned}
$$


Then,

However,

$$
\tau+F\left(d\left(x_{i+1}, x_{i+2}\right)\right) \leq F\left(m\left(x_{i}, x_{i+1}\right)\right) .
$$

$$
\begin{aligned}
m\left(x_{i}, x_{i+1}\right) & =\max \left\{d\left(x_{i}, x_{i+1}\right), d\left(x_{i}, T^{n_{i}} x_{i}\right), d\left(x_{i+1}, T^{n_{i}} x_{i+1}\right), \frac{d\left(x_{i}, T^{n_{i}} x_{i+1}\right)+d\left(x_{i+1}, T^{n_{i}} x_{i}\right)}{2}\right\} \\
& =\max \left\{d\left(x_{i}, x_{i+1}\right), d\left(x_{i+1}, x_{i+2}\right), \frac{d\left(x_{i}, x_{i+2}\right)}{2}\right\} \\
& \leq \max \left\{d\left(x_{i}, x_{i+1}\right), d\left(x_{i+1}, x_{i+2}\right), \frac{d\left(x_{i}, x_{i+1}\right)+d\left(x_{i+1}, x_{i+2}\right)}{2}\right\} \\
& \leq \max \left\{d\left(x_{i}, x_{i+1}\right), d\left(x_{i+1}, x_{i+2}\right)\right\} .
\end{aligned}
$$

If $d\left(x_{i_{0}+1}, x_{i_{0}+2}\right) \geq d\left(x_{i_{0}}, x_{i_{0}+1}\right)$ for some $i_{0} \in \mathbb{N}_{0}$, then

$$
m\left(x_{i_{0}}, x_{i_{0}+1}\right) \leq d\left(x_{i_{0}+1}, x_{i_{0}+2}\right),
$$

and since $F$ is strictly increasing,

$$
F\left(m\left(x_{i_{0}}, x_{i_{0}+1}\right)\right) \leq F\left(d\left(x_{i_{0}+1}, x_{i_{0}+2}\right)\right),
$$

so, it follows from (14) that

$$
\tau+F\left(d\left(x_{i_{0}+1}, x_{i_{0}+2}\right)\right) \leq F\left(d\left(x_{i_{0}+1}, x_{i_{0}+2}\right)\right) .
$$

So, $\tau \leq 0$, which is a contradiction. Consequently,

$$
d\left(x_{i+1}, x_{i+2}\right)<d\left(x_{i}, x_{i+1}\right), \quad \forall i \in \mathbb{N}_{0} .
$$

Hence, from (14) and (19), we have

$$
\tau+F\left(d\left(x_{i+1}, x_{i+2}\right) \leq F\left(d\left(x_{i}, x_{i+1}\right)\right)\right.
$$

or

$$
F\left(d\left(x_{i+1}, x_{i+2}\right) \leq F\left(d\left(x_{i}, x_{i+1}\right)\right)-\tau .\right.
$$

In general, one can get

$$
F\left(d\left(x_{i+1}, x_{i+2}\right) \leq F\left(d\left(x_{0}, x_{1}\right)\right)-i \tau .\right.
$$

Hence,

$$
\lim _{i \longrightarrow \infty} F\left(d\left(x_{i}, x_{i+1}\right)\right)=-\infty .
$$

So, from $\left(F_{2}\right)$, we have

$$
\lim _{i \longrightarrow \infty} d\left(x_{i}, x_{i+1}\right)=0 .
$$

Therefore, with notice to $\left(F_{3}\right)$, there exists $k \in(0,1)$ such that

$$
\lim _{i \longrightarrow \infty}\left(d\left(x_{i}, x_{i+1}\right)\right)^{k} F\left(d\left(x_{i}, x_{i+1}\right)\right)=0 .
$$

Now, (22) implies that

$$
\left(d\left(x_{i}, x_{i+1}\right)\right)^{k} F\left(d\left(x_{i}, x_{i+1}\right)\right) \leq\left(d\left(x_{i}, x_{i+1}\right)\right)^{k}\left(F\left(d\left(x_{0}, x_{1}\right)\right)-i \tau\right) \text {. }
$$

Then, it can be easily seen that

$$
\lim _{i \longrightarrow \infty} i\left(d\left(x_{i}, x_{i+1}\right)\right)^{k}=0 .
$$

So, there exists $i_{0} \in \mathbb{N}_{0}$ such that

$$
d\left(x_{i}, x_{i+1}\right) \leq \frac{1}{i^{1 / k}}, \quad \forall i \geq i_{0} .
$$

Consequently, if $m>n>n_{0}$, then

$$
\begin{aligned}
d\left(x_{n}, x_{m}\right) & \leq \sum_{j=n}^{m} d\left(x_{j}, x_{j+1}\right) \\
& \leq \sum_{j=n}^{m} \frac{1}{j^{(1 / k)}} \\
& \leq \sum_{j=n_{0}}^{\infty} \frac{1}{j^{(1 / k)}} .
\end{aligned}
$$

Since $k \in(0,1)$, the series $\sum_{j=n_{0}}^{\infty} 1 / j^{(1 / k)}$ is convergent. Therefore, $\left\{x_{i}\right\}$ is a Cauchy sequence, and since $X$ is complete, there exists $u \in X$ such that $x_{i} \longrightarrow u$ as $i \longrightarrow \infty$.As a next step, we show that $u$ is a fixed point of $T^{n(u)}$. Indeed, due to the continuity of $T$, we have

$$
d(T u, u)=\lim _{i \longrightarrow \infty} d\left(T x_{i}, x_{i}\right)=\lim _{i \longrightarrow \infty} d\left(x_{i+1}, x_{i}\right)=0,
$$

and so $u$ is a fixed point of T. For proving the uniqueness of the fixed point, let us consider $u$ and $v$ be two distinct fixed points and $n=n(u)$. So, we have $d(u, v)>0$, and hence, we get that $d(T u, T v)>0$; then, by (6) and $(\zeta 2)$,

$$
\begin{aligned}
0 & \leq \zeta\left(\tau+\alpha(u, v) F(d(T u, T v)), F\left(m(u, v)+\operatorname{LN}_{1}(u, v)\right)\right) \\
& \leq F\left(m(u, v)+L N_{1}(u, v)\right)-(\tau+\alpha(u, v) F(d(T u, T v))) .
\end{aligned}
$$

Therefore,

$$
\tau+\alpha(u, v) F(d(T u, T v)) \leq F\left(m(u, v)+\mathrm{LN}_{1}(u, v)\right) .
$$


Hence, (32) implies that

$$
\begin{aligned}
\tau+F(d(u, v)) & =\tau+F(d(T u, T v)) \\
& \leq \tau+\alpha(u, v) F(d(T u, T v)) \\
& \leq F\left(m(u, v)+\operatorname{LN}_{1}(u, v)\right) \\
& \leq F(m(u, v)+L d(u, T u)) \\
& =F(m(u, v)+0) \\
& =F(m(u, v))
\end{aligned}
$$

where

$$
\begin{aligned}
m(u, v) & =\max \left\{d(u, v), d(u, T u), d(v, T v), \frac{d(u, T v)+d(v, T u)}{2}\right\} \\
& =\max \left\{d(u, v), 0,0, \frac{d(u, v)+d(v, u)}{2}\right\} \\
& =d(u, v)
\end{aligned}
$$

So, we have

$$
\tau+F(d(u, v)) \leq F(d(u, v))
$$

which is a contradiction, as $\tau>0$. So, $u=v$.

Corollary 1. Theorem 3.3 of [7] of Theorem 4 by taking $n(x)=1$. Because in this case,

$$
\zeta\left(\tau+\alpha(x, y) F(d(T x, T y)), F\left(m(x, y)+\mathrm{LN}_{1}(x, y)\right)\right) \geq 0 .
$$

Now, by ( $\zeta 2)$, we have

$$
\begin{aligned}
0 & \leq \zeta\left(\tau+\alpha(x, y) F(d(T x, T y)), F\left(m(x, y)+\mathrm{LN}_{1}(x, y)\right)\right) \\
& \leq F\left(m(x, y)+\mathrm{LN}_{1}(x, y)\right)-(\tau+\alpha(x, y) F(d(T x, T y))) .
\end{aligned}
$$

\section{Therefore,}

$$
\tau+\alpha(x, y) F(d(T x, T y)) \leq F\left(m(x, y)+\mathrm{LN}_{1}(x, y)\right) .
$$

Corollary 2. Theorem 3 is contained in Theorem 4 by taking $m(x, y)=d(x, y), \alpha(x, y)=1$, and $L=0$. Also, Theorem 4 is reduced to theorem [8] by setting $n(x)=1$.

The following example shows that if the mapping $T$ satisfies the condition of Theorem 4 , it cannot guarantee in general the continuity of the mapping $T$.

Example 1. Let $X=\mathbb{R}$ denote the real numbers with the usual metric $d$. Define function $T: X \longrightarrow X$ by

$$
T x= \begin{cases}1, & x \in \mathbb{Q}, \\ 0, & x \in \mathbb{Q}^{c} .\end{cases}
$$

Then, $T$ discontinues at each point of $X$, and $T^{2}=1$. If $\alpha$ is an arbitrary element of $[0,1)$, then $\forall x \in X, \exists n_{x}=2 ; \quad \forall y \in X: d\left(T^{n_{x}} x, T^{n_{x}} y\right)=0 \leq \alpha d(x, y)$.

Now, it is obvious that the function $\zeta(t, s)=\alpha s-t$ of condition (6) of Theorem 4 on $[0, \infty) \times[0, \infty)$ is a simulation function and $T$ satisfies following condition:

$$
\zeta\left(d\left(T^{n} x, T^{n} y\right), d(x, y)\right) \geq 0
$$

but $T$ discontinues at each point of $X$. Moreover, $T$ satisfies all the assumptions of Theorem 4 , when $L=0$ and the unique fixed point of $T$ is $x=1$ and Picard's iteration of $T$; that is, if $y \in X$ is an arbitrary point of $X$, then $T^{n}(y)$ is convergent to the fixed point.

Theorem 5. Let $(X, d)$ be a complete metric space and $\alpha: X \times X \longrightarrow(0,+\infty)$ a symmetric function, where $\alpha(x, y) \geq 1$. Assume that $T: X \longrightarrow X$ is a mapping in which there exist $F \in \mathscr{G}, \tau>0$, and the simulation function $\zeta$ such that for all $x, y \in X$ with $T^{n(x)} x \neq T^{n(x)} y$, where $n(x)$ is a positive integer and $1 / 2 d\left(x, T^{n(x)} x\right) \leq d(x, y)$ implies

$$
\zeta\left(\tau+\alpha(x, y) F\left(d\left(T^{n(x)} x, T^{n(x)} y\right)\right), F(m(x, y))\right) \geq 0
$$

where $m(x, y)$ is defined as in Theorem 4, satisfying the following conditions:

(i) $T$ is $\alpha$-admissible,

(ii) there exists $x_{0} \in X$ such that $\alpha\left(x_{0}, T x_{0}\right) \geq 1$,

(iii) if $\left\{x_{n}\right\}$ is a sequence in $X$ such that $x_{n} \longrightarrow x$ as $n \longrightarrow \infty$ and $\alpha\left(x_{n}, x_{n+1}\right) \geq 1$ for all $n \in \mathbb{N}_{0}$, then $\alpha\left(x_{n}, x\right) \geq 1$ for all $n \in \mathbb{N}_{0}$, and

(iv) $T$ has the K-property,

then $T$ has a fixed point in $X$.

Proof. Let $x_{0} \in X$ be an arbitrary point. The recursive sequence $\left\{x_{k}\right\}$ is inductively constructed as follows: $n_{0}=n\left(x_{0}\right)$, and we set $x_{1}=T^{n_{0}} x_{0}$ and inductively get $x_{i+1}=$ $T^{n_{i}} x_{i}$ with $n_{i}=n\left(x_{i}\right)$.

We assert that $x_{i} \neq x_{i+1}$ for all $i \in \mathbb{N}_{0}$. Suppose, on the contrary, that there exists $i_{0} \in N_{0}$ such that $x_{i_{0}}=x_{i_{0}+1}=T^{n_{i_{0}}} x_{i_{0}}$. Then, $x_{i_{0}}$ turns to be a fixed point of $T^{n_{i_{0}}}$. On the other hand,

$$
T x_{i_{0}}=T\left(T^{n_{i_{0}}} x_{i_{0}}\right)=T^{n_{i_{0}}}\left(T x_{i_{0}}\right) .
$$

Thus, $T x_{i_{0}}$ form a fixed point of $T^{n_{i_{0}}}$. If $T x_{i_{0}}=x_{i_{0}}$, then we conclude that $T$ has a fixed point and that terminate the proof. Suppose, on the contrary, that $T x_{i_{0}} \neq x_{i_{0}}$ and hence $d\left(T^{n_{i_{0}}}\left(T x_{i_{0}}\right), T^{n_{i_{0}}}\left(x_{i_{0}}\right)\right)>0$. Then, by (42), we have

$$
\begin{aligned}
0 & \leq\left(\tau+\alpha\left(x_{i_{0}}, T x_{i_{0}}\right) F\left(d\left(T^{n_{i_{0}}} x_{i_{0}}, T^{n_{i_{0}}} T x_{i_{0}}\right)\right), F\left(m\left(x_{i_{0}}, T x_{i_{0}}\right)\right),\right. \\
& \leq F\left(m\left(x_{i_{0}}, T x_{i_{0}}\right)\right)-\left(\tau+\alpha\left(x_{i_{0}}, T x_{i_{0}}\right) F\left(d\left(T^{n_{i_{0}}} x_{i_{0}}, T^{n_{i_{0}}} T x_{i_{0}}\right)\right)\right) .
\end{aligned}
$$

Hence, 


$$
\begin{array}{rlr}
\tau+F\left(d\left(x_{i_{0}}, T x_{i_{0}}\right)\right) & =\tau+F\left(d\left(T^{n_{i_{0}}} x_{i_{0}}, T^{n_{i_{0}}} T x_{i_{0}}\right)\right) & \text { However }, \\
& \leq \tau+\alpha\left(x_{i_{0}}, T x_{i_{0}}\right) F\left(d\left(T^{n_{i_{0}}} x_{i_{0}}, T^{n_{i_{0}}} T x_{i_{0}}\right)\right) \\
& \leq F\left(m\left(x_{i_{0}}, T x_{i_{0}}\right)\right) . &
\end{array}
$$

$$
\begin{aligned}
m\left(x_{i_{0}}, T x_{i_{0}}\right) & =\max \left\{d\left(x_{i_{0}}, T x_{i_{0}}\right), d\left(x_{i_{0}}, T^{n_{i_{0}}} x_{i_{0}}\right), d\left(T x_{i_{0}}, T^{n_{i_{0}}} T x_{i_{0}}\right), \frac{d\left(x_{i_{0}}, T^{n_{i_{0}}} T x_{i_{0}}\right)+d\left(T x_{i_{0}}, T^{n_{i_{0}}} x_{i_{0}}\right)}{2}\right\} \\
& =\left\{d\left(x_{i_{0}}, T x_{i_{0}}\right)\right\} .
\end{aligned}
$$

Therefore,

$$
\tau+F\left(d\left(x_{i_{0}}, T x_{i_{0}}\right)\right) \leq F\left(d\left(x_{i_{0}}, T x_{i_{0}}\right)\right) .
$$

So, $\tau \leq 0$, which is a contradiction. Consequently, we deduce that, for all $i \in \mathbb{N}_{0}, x_{i} \neq x_{i+1}$. Then, $d\left(x_{i+1}, x_{i}\right)>0$, and so

$$
\frac{1}{2} d\left(x_{i}, T^{n_{i}} x_{i}\right)=\frac{1}{2} d\left(x_{i}, x_{i+1}\right) \leq d\left(x_{i}, x_{i+1}\right) .
$$

Now, by (42),

$$
\begin{aligned}
\tau+F\left(d\left(x_{i+1}, x_{i+2}\right)\right) & =\tau+F\left(d\left(T^{n_{i}} x_{i}, T^{n_{i}} x_{i+1}\right)\right. \\
& \leq \tau+\alpha\left(x_{i}, x_{i+1}\right) F\left(d\left(T^{n_{i}} x_{i}, T^{n_{i}} x_{i+1}\right)\right. \\
& \leq F\left(m\left(x_{i}, x_{i+1}\right)\right) .
\end{aligned}
$$

Hence,

$$
\tau+F\left(d\left(x_{i+1}, x_{i+1}\right)\right) \leq F\left(m\left(x_{i}, x_{i+1}\right)\right) .
$$

However,

$$
\begin{aligned}
m\left(x_{i}, x_{i+1}\right) & =\max \left\{d\left(x_{i}, x_{i+1}\right), d\left(x_{i}, T^{n_{i}} x_{i}\right), d\left(x_{i+1}, T^{n_{i}} x_{i+1}\right), \frac{d\left(x_{i}, T^{n_{i}} x_{i+1}\right)+d\left(x_{i+1}, T^{n_{i}} x_{i}\right)}{2}\right\} \\
& =\max \left\{d\left(x_{i}, x_{i+1}\right), d\left(x_{i+1}, x_{i+2}\right), \frac{d\left(x_{i}, x_{i+2}\right)}{2}\right\} \\
& \leq \max \left\{d\left(x_{i}, x_{i+1}\right), d\left(x_{i+1}, x_{i+2}\right), \frac{d\left(x_{i}, x_{i+1}\right)+d\left(x_{i+1}, x_{i+2}\right)}{2}\right\} \\
& \leq \max \left\{d\left(x_{i}, x_{i+1}\right), d\left(x_{i+1}, x_{i+2}\right)\right\} .
\end{aligned}
$$

If $d\left(x_{i_{0}+1}, x_{i_{0}+2}\right) \geq d\left(x_{i_{0}}, x_{i_{0}+1}\right)$ for some $i_{0} \in \mathbb{N}_{0}$, then

$$
m\left(x_{i_{0}}, x_{i_{0}+1}\right) \leq d\left(x_{i_{0}+1}, x_{i_{0}+2}\right),
$$

and since $F$ is strictly increasing,

$$
F\left(m\left(x_{i_{0}}, x_{i_{0}+1}\right)\right) \leq F\left(d\left(x_{i_{0}+1}, x_{i_{0}+2}\right)\right),
$$

so, it follows from (50) that

$$
\tau+F\left(d\left(x_{i_{0}+1}, x_{i_{0}+2}\right)\right) \leq F\left(d\left(x_{i_{0}+1}, x_{i_{0}+2}\right)\right) .
$$

Hence, $\tau \leq 0$, which is a contradiction. Therefore,

$$
d\left(x_{i+1}, x_{i+2}\right)<d\left(x_{i}, x_{i+1}\right), \quad \forall i \in \mathbb{N}_{0} .
$$

Hence, from (50) and (55), we have

$$
\tau+F\left(d\left(x_{i+1}, x_{i+2}\right) \leq F\left(d\left(x_{i}, x_{i+1}\right)\right)\right.
$$

or

$$
F(d)\left(x_{i+1}, x_{i+2}\right) \leq F\left(d\left(x_{i}, x_{i+1}\right)\right)-\tau .
$$

Consequently,

$$
F\left(d\left(x_{i+1}, x_{i+2}\right) \leq F\left(d\left(x_{0}, x_{1}\right)\right)-i \tau .\right.
$$

Hence,

$$
\lim _{i \longrightarrow \infty} F\left(d\left(x_{i}, x_{i+1}\right)\right)=-\infty \text {. }
$$

So, from $\left(G_{2}\right)$, we have

$$
\lim _{i \longrightarrow \infty} d\left(x_{i}, x_{i+1}\right)=0 .
$$

Now, we claim that $\left\{x_{i}\right\}$ is a Cauchy sequence. If it is not true, then by Lemma 2, there exist $\varepsilon_{0}>0$ and two sequences 
of positive integers $\left\{n_{k}\right\}$ and $\left\{m_{k}\right\}$ with $n_{k}>m_{k}>k$ such that $d\left(x_{m_{k}}, x_{n_{k}}\right)>\epsilon_{0}, d\left(x_{m_{k}}, x_{n_{k}-1}\right)<\varepsilon_{0}$, and
(L1) $\lim _{k \longrightarrow \infty} d\left(x_{n_{k}}, x_{m_{k}}\right)=\varepsilon_{0}$
(L2) $\lim _{k \longrightarrow \infty} d\left(x_{n_{k}}, x_{m_{k}-1}\right)=\varepsilon_{0}$

(L3) $\lim _{k \longrightarrow \infty} d\left(x_{n_{k}+1}, x_{m_{k}}\right)=\varepsilon_{0}$

(L4) $\lim _{k \rightarrow \infty} d\left(x_{n_{k}+1}, x_{m_{k}-1}\right)=\varepsilon_{0}$

Therefore, the definition of $m(x, y)$ implies

$$
\begin{aligned}
\lim _{k \longrightarrow \infty} m\left(x_{n_{k}}, x_{m_{k}-1}\right) & =\lim _{k \longrightarrow \infty} \max \left\{d\left(x_{n_{k}}, x_{m_{k}-1}\right), d\left(x_{n_{k}}, T^{n(x)} x_{n_{k}}\right), d\left(x_{m_{k}-1}, T^{n(x)} x_{m_{k}-1}\right), \frac{d\left(x_{n_{k}}, T^{n(x)} x_{m_{k}-1}\right)+d\left(x_{m_{k}-1}, T^{n(x)} x_{n_{k}}\right)}{2}\right\} \\
& =\lim _{k \longrightarrow \infty} \max \left\{d\left(x_{n_{k}}, x_{m_{k}-1}\right), d\left(x_{n_{k}}, x_{n_{k}+1}\right), d\left(x_{m_{k}-1}, x_{m_{k}}\right), \frac{d\left(x_{n_{k}}, x_{m_{k}}\right)+d\left(x_{m_{k}-1}, x_{n_{k}+1}\right)}{2}\right\} \\
& =\max \left\{\varepsilon_{0}, 0,0, \frac{\varepsilon_{0}+\varepsilon_{0}}{2}\right\}=\varepsilon_{0} .
\end{aligned}
$$

So,

$$
\lim _{k \longrightarrow \infty} m\left(x_{n_{k}}, x_{m_{k}-1}\right)=\varepsilon_{0} .
$$

On the other hand, since $\lim _{k \longrightarrow \infty} d\left(x_{n_{k}}, x_{m_{k}-1}\right)=\varepsilon_{0}>0$ and $\lim _{k \rightarrow \infty} d\left(x_{n_{k}}, x_{n_{k}+1}\right)=0$, with considering a subsequence if it is needed, one can suppose that there exists $k_{1} \in \mathbb{N}$ such that for any $k>k_{1}$ and $n_{k}>m_{k}>k$,

$$
d\left(x_{n_{k}}, x_{n_{k}+1}\right) \leq d\left(x_{n_{k}}, x_{m_{k}-1}\right) \text {. }
$$

So, it is obvious that, for all $k>k_{1}$ and $n_{k}>m_{k}>k$,

$$
\frac{1}{2} d\left(x_{n_{k}}, T^{n(x)} x_{n_{k}}\right)=\frac{1}{2} d\left(x_{n_{k}}, x_{n_{k}+1}\right)<d\left(x_{n_{k}}, x_{m_{k}-1}\right) .
$$

Also, using the K-property, there exists $k_{2} \in \mathbb{N}$ such that

$$
\alpha\left(x_{n_{k}}, x_{m_{k}-1}\right) \geq 1, \quad \forall k>k_{2} .
$$

If $k \geq \max \left\{k_{1}, k_{2}\right\}$, then it follows from (65) that

$$
\begin{aligned}
\tau+F\left(d\left(T^{n(k)} x_{n_{k}}, x_{m_{k}-1}\right)\right) \leq & \tau+\alpha\left(x_{n_{k}}, x_{m_{k}-1}\right) F \\
& \cdot\left(d\left(T^{n(x)} x_{n_{k}}, T^{n(x)} x_{m_{k}-1}\right)\right) \\
\leq & F\left(m\left(x_{n_{k}}, x_{m_{k}-1}\right)\right) .
\end{aligned}
$$

Letting $n \longrightarrow \infty$, the continuity of $F$ through (L1) and (62) implies

$$
\tau+F\left(\varepsilon_{0}\right) \leq F\left(\varepsilon_{0}\right),
$$

which is contradicted by $\tau>0$. Consequently, $\left\{x_{i}\right\}$ is a Cauchy sequence in the complete metric space $X$. Hence, there exists $u \in X$ such that $x_{i} \longrightarrow u$, as $n \longrightarrow \infty$. To complete the proof, we show that $u$ is a fixed point of $T$. We first claim, for all $n \geq 0$, that

$$
\frac{1}{2} d\left(x_{i}, x_{i+1}\right) \leq d\left(x_{i}, u\right) \text {, or } \frac{1}{2} d\left(x_{i+1}, x_{i+2}\right) \leq d\left(x_{i+1}, u\right) \text {. }
$$

In fact, if we assume that, for some $i_{0} \geq 0$, both of them are false, then

$$
\frac{1}{2} d\left(x_{i_{0}}, x_{i_{0}+1}\right)>d\left(x_{i_{0}}, u\right) \text {, and } \frac{1}{2} d\left(x_{i_{0}+1}, x_{i_{0}+2}\right)>d\left(x_{i_{0}+1}, u\right) \text {. }
$$

Hence, (55) implies

$$
\begin{aligned}
d\left(x_{i_{0}}, x_{i_{0}+1}\right) & \leq d\left(x_{i_{0}}, u\right)+d\left(u, x_{i_{0}+1}\right) \\
& <\frac{1}{2} d\left(x_{i_{0}}, x_{i_{0}+1}\right)+\frac{1}{2} d\left(x_{i_{0}+1}, x_{i_{0}+2}\right) \\
& \leq \frac{1}{2} d\left(x_{i_{0}}, x_{i_{0}+1}\right)+\frac{1}{2} d\left(x_{i_{0}}, x_{i_{0}+1}\right) \\
& =d\left(x_{i_{0}}, x_{i_{0}+1}\right)
\end{aligned}
$$

which is a contradiction and the claim is proved.

Now, let us begin with the first part of (68); that is, suppose that

$$
\frac{1}{2} d\left(x_{i}, x_{i+1}\right) \leq d\left(x_{i}, u\right)
$$

and on the contrary, assume that $T u \neq u$. Without loss of generality, one can imagine that $T x_{i} \neq T u$, for all $i \in \mathbb{N}_{0}$ (because if $x_{i+1}=T x_{i}=T u$ for infinite values of $i$, then uniqueness of the limit concludes that $T u=u$ ). Then, from (45) and (iii), we get

$$
\begin{aligned}
\tau+F\left(d\left(x_{i+1}, T u\right)\right) & =\tau+F\left(d\left(T x_{i}, T u\right)\right) \\
& \leq \tau+\alpha\left(x_{i}, u\right) F\left(d\left(T x_{i}, T u\right)\right) \\
& \leq F\left(m\left(x_{i}, u\right)\right) .
\end{aligned}
$$

And since $F$ is continuous on $(0,+\infty)$, and $d(u, T u)>0$, as $i \longrightarrow \infty$, we get

$$
\tau+F(d(u, T u)) \leq F\left(\lim _{i \longrightarrow \infty}\left(m\left(x_{i}, u\right)\right) .\right.
$$

However, 


$$
\begin{aligned}
m\left(x_{i}, u\right)= & \max \left\{d\left(x_{i}, u\right), d\left(x_{i}, x_{i+1}\right), d(u, T u),\right. \\
& \left.\frac{d\left(x_{i}, T u\right)+d\left(u, x_{i+1}\right)}{2}\right\} .
\end{aligned}
$$

So, we have

$$
\begin{aligned}
\lim _{i \rightarrow \infty} m\left(x_{i}, u\right) & =\max \left\{0,0, d(u, T u), \frac{d(u, T u)+0}{2}\right\} \\
& =d(u, T u) .
\end{aligned}
$$

Therefore, if $d(u, T u) \neq 0$, then from (73), we have

$$
\tau+F(d(u, T u)) \leq F(d(u, T u))
$$

which is contradicted, as $\tau>0$. So, $d(u, T u)=0$, i.e., $T u=u$. Finally, if we assume that the second part of (68) is true, i.e.,

$$
\frac{1}{2} d\left(x_{i+1}, x_{i+2}\right) \leq d\left(x_{i+1}, u\right)
$$

then by using the same manner, we can prove that $d(u, T u)=0$, i.e., $T u=u$.

Suppose that $u$ and $v$ are two fixed points of $T$. If $u \neq v$, then $d(T u, T v)>0$. Furthermore, $\alpha(u, v) \geq 1$, because $u, v \in \operatorname{Fix}(T)$. It is also clear that $1 / 2 d(u, T u)=0<d(u, v)$. Hence, (45) implies

$$
\begin{aligned}
\tau+F(d(u, v)) & =\tau+F(d(T u, T v)) \\
& \leq \tau+\alpha(u, v) F(d(T u, T v)) \\
& \leq F(m(u, v))
\end{aligned}
$$

where

$$
\begin{aligned}
m(u, v) & =\max \left\{d(u, v), d(u, T u), d(v, T v), \frac{d(u, T v)+d(v, T u)}{2}\right\} \\
& =\max \left\{d(u, v), 0,0, \frac{d(u, v)+d(v, u)}{2}\right\} \\
& =d(u, v)
\end{aligned}
$$

So, we get

$$
\tau+F(d(u, v)) \leq F(d(u, v))
$$

which is a contradicted by $\tau>0$ and so $u=v$. This completes the proof.

Corollary 3. If in Theorem 5, we put $n(x)=1$, then

$$
\zeta(\tau+\alpha(x, y) F(d(T x, T y)), F(m(x, y))) \geq 0 .
$$

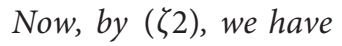

$$
\begin{aligned}
0 & \leq \zeta(\tau+\alpha(x, y) F(d(T x, T y)), F(m(x, y))) \\
& \leq F(m(x, y))-(\tau+\alpha(x, y) F(d(T x, T y))) .
\end{aligned}
$$

Therefore,

$$
\tau+\alpha(x, y) F(d(T x, T y)) \leq F(m(x, y)) .
$$

Hence, we get Theorem 3.3 of [7].

\section{Data Availability}

The data used to support the findings of this study are included within the article.

\section{Conflicts of Interest}

The authors declare that they have no conflicts of interest.

\section{Acknowledgments}

The paper was supported by the National Natural Science Foundation of China (no. 11671365).

\section{References}

[1] S. Banach, "Sur les opérations dans les ensembles abstraits et leur application aux équations intégrales," Fundamenta Mathematicae, vol. 3, pp. 133-181, 1922.

[2] V. W. Bryant, "A remark on a fixed-point theorem for iterated mappings," The American Mathematical Monthly, vol. 75, no. 4, pp. 399-400, 1968.

[3] F. Khojasteh, S. Shukla, and S. Radenovic, "A new approach to the study of fixed point theory for simulation functions," Filomat, vol. 29, no. 6, pp. 1189-1194, 2015.

[4] A. Öztürk, "A fixed point theorem for mappings with a F-contractive iterate," Advances in the Theory of Nonlinear Analysis and its Application, vol. 3, no. 3, pp. 231-235, 2019.

[5] H. Piri and P. Kumam, "Some fixed point theorems concerning F-contraction in complete metric spaces," Fixed Point Theory and Applications, vol. 2014, p. 210, 2014.

[6] B. Samet, C. Vetro, and P. Vetro, "Fixed point theorems for -contractive type mappings," Nonlinear Analysis: Theory, Methods \& Applications, vol. 75, no. 4, pp. 2154-2165, 2012.

[7] A. Taheri and A. P. Farajzadeh, "A new generalization of \$lalpha-type almost-\$F\$-contractions and \$lalpha-type \$F\$-Suzuki contractions in metric spaces and their fixed point theorems," Carpathian Mathematical Publications, vol. 11, no. 2, pp. 475-492, 2019.

[8] D. Wardowski, "Fixed points of a new type of contractive mappings in complete metric spaces," Fixed Point Theory and Applications, vol. 2012, p. 94, 2012.

[9] L.-C. Ceng, A. Petrusel, A. Petruşel, J.-C. Yao, and Y. Yao, "Hybrid viscosity extragradient method for systems of variational inequalities, fixed Points of nonexpansive mappings, zero points of accretive operators in Banach spaces," Fixed Point Theory, vol. 19, no. 2, pp. 487-502, 2018.

[10] Y. Yao, L. Leng, M. Postolache, and X. Zheng, "Mann-type iteration method for solving the split common fixed point problem," Journal of Nonlinear and Convex Analysis, vol. 18, pp. 875-882, 2017.

[11] Y. Yao, M. Postolache, and J. C. Yao, "An iterative algorithm for solving the generalized variational inequalities and fixed points problems," Mathematics, vol. 7, p. 61, 2019.

[12] H. Zegeye, N. Shahzad, and Y. Yao, "Minimum-norm solution of variational inequality and fixed point problem in Banach spaces," Optimization, vol. 64, no. 2, pp. 453-471, 2015. 
[13] N. Shahzad and H. Zegeye, "Convergence theorems of common solutions for fixed point, variational inequality and equilibrium problems," Journal of Nonlinear and Variational Analysis, vol. 3, pp. 189-203, 2019.

[14] B. Tan, S. Li, and S. Y. Cho, "Strong convergence of inertial Mann algorithms for solving hierarchical fixed point problems," Journal of Nonlinear and Variational Analysis, vol. 4, pp. 337-355, 2020.

[15] L.-C. Ceng, A. Petrusel, A. Petruşel, J.-C. Yao, and Y. Yao, "Systems of variational inequalities with hierarchical variational inequality constraints for Lipschitzian pseudocontractions," Fixed Point Theory, vol. 20, no. 1, pp. 113-134, 2019.

[16] Y. Yao, Y. C. Liou, and J. C. Yao, "Split common fixed point problem for two quasi-pseudocontractive operators and its algorithm construction," Fixed Point Theory and Applications, vol. 2015, p. 127, 2015.

[17] Y. Yao, Y.-C. Liou, and J.-C. Yao, "Iterative algorithms for the split variational inequality and fixed point problems under nonlinear transformations," The Journal of Nonlinear Sciences and Applications, vol. 10, no. 2, pp. 843-854, 2017.

[18] Y. Yao, X. Qin, and J. C. Yao, "Projection methods for firmly type nonexpansive operators," Journal of Nonlinear and Convex Analysis, vol. 19, pp. 407-415, 2018.

[19] V. M. Sehgal, "A fixed point theorem for mappings with a contractive iterate," Proceedings of the American Mathematical Society, vol. 23, no. 3, p. 631, 1969.

[20] NA. Secelean, "Iterated function systems consisting of F-contractions," Fixed Point Theory and Applications, vol. 2013, p. 277, 2013.

[21] G. Heidary Joonaghany, A. Farajzadeh, M. Azhini, and F. Khojasteh, "A new common fixed point theorem for Suzuki type contractions via generalized $\Psi$-simulation functions," Sahand Communications in Mathematical Analysis, vol. 16, no. 1, pp. 129-148, 2019.

[22] A. Farajzadeh, C. Noytaptim, and A. Kaewcharoen, "Some fixed point theorems for generalized-geraghty contractive type mappings in partial-metric spaces," Journal of Informatics and Mathematical Sciences, vol. 10, no. 3, pp. 455-578, 2018.

[23] H. Alikhani, D. Gopal, M. A. Miandaragh, S. Rezapour, and N. Shahzad, "Some endpoint results for $\beta$-generalized weak contractive multifunctions," Scientific World Journal, vol. 2013, Article ID 948472, 2013.

[24] B. S. Choudhury, P. Konar, B. E. Rhoades, and N. Metiya, "Fixed point theorems for generalized weakly contractive mappings," Nonlinear Analysis: Theory, Methods \& Applications, vol. 74, no. 6, pp. 2116-2126, 2011. 